\title{
Both carboxy-terminus NES motif and mutated tryptophan(s) are crucial for aberrant nuclear export of nucleophosmin leukemic mutants in $\mathrm{NPMc}^{+} \mathrm{AML}$
}

\author{
Brunangelo Falini, Niccolò Bolli, Jing Shan, Maria Paola Martelli, Arcangelo Liso, Alessandra Pucciarini, Barbara Bigerna, \\ Laura Pasqualucci, Roberta Mannucci, Roberto Rosati, Paolo Gorello, Daniela Diverio, Giovanni Roti, Enrico Tiacci, \\ Giovanni Cazzaniga, Andrea Biondi, Suzanne Schnittger, Torsten Haferlach, Wolfgang Hiddemann, Massimo F. Martelli, \\ Wei Gu, Cristina Mecucci, and Ildo Nicoletti
}

\begin{abstract}
We recently identified aberrant cytoplasmic expression of nucleophosmin (NPM) as the immunohistochemical marker of a large subgroup of acute myeloid leukemia (AML) (about one-third of adult AML) that is characterized by normal karyotype and mutations occurring at the exon-12 of the NPM gene. In this paper, we have elucidated the molecular mechanism underlying the abnormal cytoplasmic localization of NPM. All 29 AMLassociated mutated NPM alleles so far identified encode abnormal proteins which have
\end{abstract}

acquired at the $\mathrm{C}$-terminus a nuclear export signal (NES) motif and lost both tryptophan residues 288 and 290 (or only the residue 290) which determine nucleolar localization. We show for the first time that both alterations are crucial for NPM mutant export from nucleus to cytoplasm. In fact, the cytoplasmic accumulation of NPM is blocked by leptomycin-B and ratjadones, specific exportin-1/Crm1-inhibitors, and by reinsertion of tryptophan residues 288 and 290, which respectively relocate NPM mutants in the nucleoplasm and nucleoli. NPM leukemic mutants in turn recruit the wild-type NPM from nucleoli to nucleoplasm and cytoplasm. These findings indicate that potential therapeutic strategies aimed to retarget NPM to its physiological sites will have to overcome 2 obstacles, the new NES motif and the mutated tryptophan(s) at the NPM mutant C-terminus. (Blood. 2006;107: 4514-4523)

() 2006 by The American Society of Hematology

\section{Introduction}

In acute myeloid leukemia (AML), a clinically and molecularly heterogeneous disease, ${ }^{1}$ recurrent cytogenetic abnormalities help define subgroups with different prognosis, and identify patients who might benefit from targeted therapies. ${ }^{1}$ However, almost half adult AMLs display normal karyotype at conventional cytogenetics, ${ }^{2}$ and the clinical and molecular features of this large subgroup of patients are still poorly understood. ${ }^{3-7}$

We recently observed that about $60 \%$ of adult AML with normal karyotype display aberrant cytoplasmic expression of nucleophosmin (NPM) ${ }^{8}$ A multifunctional protein ${ }^{9-15}$ that characteristically shuttles between the nucleus and the cytoplasm, ${ }^{16}$ NPM is found mainly in the nucleolus, ${ }^{17-19}$ where it is one of the most abundant of the approximately 700 proteins so far identified by proteomic techniques. ${ }^{20}$ Cytoplasmic NPM identifies a distinct subgroup of AML, named $\mathrm{NPMc}^{+} \mathrm{AML}$, that accounts for about $35 \%$ of all adult AML and is characterized by wide morphologic spectrum, multilineage involvement, high frequency of FLT3-ITD mutations, absence of $\mathrm{CD} 34$, and relatively good response to induction therapy. ${ }^{8} \mathrm{NPMc}^{+}$AML also has a distinct gene expression pro- file $^{21}$ and carries mutations in exon-12 of the NPM gene ${ }^{8}$ that serve as predictor of favorable prognosis in AML with normal karyotype, ${ }^{22-24}$ and as a marker for monitoring of minimal residual disease. ${ }^{25}$

In spite of the close association between the aberrant cytoplasmic expression of NPM and exon-12 NPM mutations, ${ }^{8}$ the mechanism underlying cytoplasmic accumulation of NPM in leukemic cells and its interference with wild-type NPM protein remained to be elucidated.

Recently, Nakagawa et $\mathrm{a}^{26}$ asserted that the creation of a nuclear export signal (NES) motif at the C-terminus of NPM leukemic mutants (as consequence of the exon-12 NPM mutations) is alone responsible for cytoplasmic dislocation of NPM. In this paper, we provide evidence that the new NES motif created by the mutational event is not in itself sufficient to cause nuclear export of the NPM leukemic mutants and demonstrate it needs to act in concert with the mutated tryptophan(s) 288 and 290 at the mutant C-terminus. These findings have a relevant biological and potentially therapeutic significance.
From the Institute of Hematology and Internal Medicine, University of Perugia, Italy; the Institute for Cancer Genetics and Department of Pathology, Columbia University, New York, NY; the Institute of Hematology, University of Bari, Italy; the Institute of Hematology, University of Foggia, Italy; the Institute of Hematology, University La Sapienza, Rome, Italy; the Pediatric Clinic, M. Tettamanti Research Center, San Gerardo Hospital, Monza, Italy; and the Laboratory for Leukemia Diagnostics, Department of Internal Medicine III, Ludwig-Maximilian University, Munich, Germany.

Submitted November 30, 2005; accepted January 21, 2006. Prepublished online as Blood First Edition Paper, February 2, 2006; DOI 10.1182/blood2005-11-4745.

Supported by the Associazione Italiana per la Ricerca sul Cancro (AIRC). L.P. is a Special Fellow of the Leukemia and Lymphoma Society. N.B. is supported by the Federazione Italiana per la Ricerca sul Cancro (FIRC).
Two of the authors (B.F. and C.M.) have applied for a patent, related to the work described in the present study, on the clinical use of NPM mutants.

N.B. and J.S. contributed equally to this work.

The online version of this article contains a data supplement.

An Inside Blood analysis of this article appears at the front of this issue.

Reprints: Brunangelo Falini, Institute of Hematology, Policlinico Monteluce, 06122 Perugia, Italy; e-mail: faliniem @ unipg.it.

The publication costs of this article were defrayed in part by page charge payment. Therefore, and solely to indicate this fact, this article is hereby marked "advertisement" in accordance with 18 U.S.C. section 1734.

(C) 2006 by The American Society of Hematology 


\section{Materials and methods}

\section{Cells for transfection, primary AML samples, and OCI/AML3 cell line}

Transfections were performed on NIH-3T3 murine fibroblasts and human lung carcinoma H1299 cells. Lymphoprep-isolated leukemic cells for biochemical studies were obtained from 3 patients with AML carrying NPM mutation A and 2 patients with AML with wild-type NPM. Bone marrow trephines from 20 patients with AML (10 with NPM mutations and 10 without NPM mutations) were B5-fixed/decalcified and embedded in paraffin, as previously described. ${ }^{8}$ The 10 NPM-mutated cases included 7 mutations A, 1 mutation $\mathrm{B}$, and 2 mutations D. The human AML cell line OCI/AML3, which carries an exon-12 NPM mutation A, ${ }^{27}$ was provided by H. Drexler and grown in alpha-modified Eagle medium (MEM) plus 10\% fetal bovine serum (FBS), $1 \%$ glutamine, and antibiotics.

\section{Plasmid constructs}

NPM mutants A, B, C, and D were generated by polymerase chain reaction (PCR) using NPMwt as template; the same forward primer (5'CGCCACGCTAGCGAAGATTCGATGGAC-3') with different reverse primers were used for each mutant (mutant A: 5'-CTATTTTCTTAAAGAGACTTCCTCCACTGCCAGACAGAGATCTTGAATAGCCTCTTGG-3'; mutant B:5'-CTATTTTCTTAAAGAGACTTCCTCCACTGCCATGCAGAGATCTTGAAT AGCCTCTTGG; mutant C: $5^{\prime}$-CTATTTTCTTAAAGAGACTTCCTCCACTGCCACGCA GAGATCTTGAATAGCCTCTTGG; and mutant D: 5-CTATTTTCTTAAAGAGACTTCCTCCAC TGCCAGGCAGAGATCTTGAATAGCCTCTTGG). The PCR products of mutants A, B, C, and D were cloned into the pcDNA3.1/NT-GFP-TOPO vector (Invitrogen, Carlsbad, CA). To generate the double-flag-HA tag at the N-terminal of both wild-type NPM and mutant A, PCR was performed with either wild-type or mutant A as template; 5'-CGCCACGCTAGCGAAGATTCGATGGAC and 5'-TCAAGAATTCCAGAAATGAAATAAGACGG were used, respectively, as forward and reverse primers. The PCR product was excised by NheI and EcoRI, and the resulting fragment was subcloned into the PCIN4 vector containing the Flag-HA tag at the $\mathrm{N}$-terminal of the inserted fragment.

Constructs pEGFP-C1-NPM_MUT_A_A290W, pEGFP-C1NPM_MUT_A_C288W + A290W, and pEGFP-C1-NPM_MUT_A_NO_ 2nd_NES were generated using pEGFP-C1-NPMmA ${ }^{8}$ as a template, by a set of 2 partially self-complementary primers bearing the desired mutation. The 2 primers were annealed, and the resulting double-stranded DNA sequence bearing $B g l I I-E c o$ RI-compatible ends was ligated into the pEGFP-C1-NPMmA vector previously digested with the same 2 enzymes, exploiting the localization of the mutated region between these restriction sites. The sequences of primers are the following: NPM MUT_A_A290W_FOR: 5'-GATCTCTGTCTGTGGGTGGAGGAAGT-

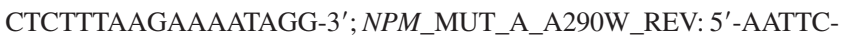
CTATTTTCTTAAAGAGACTTCCTCCACCCACAGACAGA-3'; NPM _MUT_A_C288W+A290W_FOR: 5'-GATCTCTGGCTGTGGGTGGAGGAAGTCTCTTTAAGAAAATAGG-3'; NPM_MUT_A_C288W+A290W_ REV: 5'-AATTCCTATTTTCTTAAAGAGACTTCCTCCACCCACAGCCAGA-3'; NPM_MUT_A_NO_2nd_NES_FOR: 5'-GATCTCTGTGGAGCAGGGGAGGAAGGCTCTTTAAGAAAATAGG-3'; and NPM_MUT_A_ NO 2nd NES_REV: 5'-AATTCCTATTTTCTTAAAGAGCCTTCCTCCCCTGCTCCACAGA-3'.

pEGFP-C1-NPM mutants E, G, and pEGFP-C1-NPM_MUT_ A_NO_1st_NES were generated with the QuikChange Multi Site-Directed Mutagenesis Kit (Stratagene, La Jolla, CA), using pEGFP-C1-NPMwt ${ }^{8}$ (for mutants $\mathrm{E}$ and $\mathrm{G}$ ) and pEGFP-C1-NPMmA (for deletion of the first NES) as a template. The manufacturer's instructions were followed except for transformation of bacteria, which was done with $5 \mu \mathrm{L}$ PCR products instead of $1 \mu \mathrm{L}$. Primers were designed on the following sequences: $N P M$ _MUT_E: 5'-GATCTCTGGCAGTCTCTTGCCCAAGTCTCTTTAAG3'; NPM_MUT_G: 5'-GATCTCTGGCAGTGCTTCGCCCAAGTCTCTTTAAG-3'; and NPM_MUT_A_NO_1st_NES: 5'-GCACCAGT-
TATCTGGAAGAACGGGCAGTTTAGGGGCTGG-3' . Every construct was validated by DNA sequencing.

\section{Antibodies}

We previously used anti-NPM monoclonal antibodies ${ }^{17,18}$ that recognize both wild-type and mutated NPM proteins. To study the NPM mutants without the interference of wild-type NPM, we generated an affinity-purified polyclonal antibody (Sil-C) against the synthetic peptide $\mathrm{NHCOCH}_{3}$ CLAVEEVSLRK-COOH (Inbio-Ltd, Tallin, Estonia) corresponding to NPM mutant A. The specificity of Sil-C for the NPM mutant was confirmed by Western blotting (Figure S1, available on the Blood website; see the Supplemental Figure link at the top of the online article). To assess the subcellular distribution of wild-type NPM without the interference of leukemic NPM mutants, we used a mouse monoclonal antibody (clone FC-61991) that recognizes the wild-type but not mutant NPM proteins (Invitrogen, Carlsbad, CA). The antinucleolin/C23 monoclonal antibody, used as a control, was purchased from DakoCytomation (Glostrup, Denmark).

\section{Inhibition of the Crm1-dependent nuclear export pathway}

Expression vectors $(5 \mu \mathrm{g})$ coding HA-NPM wild-type, enhanced green fluorescent protein (eGFP)-NPMmA, or both were transfected in H1299 cells by the calcium-phosphate precipitation method. After 24 hours, cells were treated with $20 \mathrm{nM}$ leptomycin B (LMB; Sigma, St Louis, MO) for 8 hours or left untreated. Cells were fixed with $4 \%$ paraformadehyde for immunofluorescence analysis.

NIH-3T3 cells were transfected using Lipofectamine 2000 (Invitrogen) following manufacturer's instructions. After 24 hours, cells grown on glass coverslips were incubated with $10 \mu \mathrm{g} / \mathrm{mL}$ cycloheximide (Merck Biosciences, Nottingham, United Kingdom) (30 minutes), and $20 \mathrm{ng} / \mathrm{mL}$ LMB (Merck Biosciences) (5 hours) or $20 \mathrm{ng} / \mathrm{mL}$ ratjadones A and C (Alexis Biochemicals, Carlsbad, CA) (5 hours). For immunofluorescence studies, cells were fixed in $4 \%$ paraformaldehyde (10 minutes). For time-course experiments, transfected cells were transferred on an Attofluor chamber (Molecular Probes, Eugene, OR) and observed on a MRC-1024 (BioRad, Cambridge, United Kingdom) confocal apparatus mounted on an Olympus IMT-2 microscope with an SPlan $60 \times / 1.4$ numeric aperture (NA) oil objective (Olympus, Tokyo, Japan). Images of a single slice of cells were recorded in the basal state and after LMB addition at 60-second intervals using the time-series function of the LaserSharp (BioRad) software. The 488-nm line of an argon laser was used for excitation and a 505-550-nm band-pass filter on the PMT2 was used for collecting the fluorescence emission. The images were processed and analyzed with the public-domain ImageJ software (Rasband WS, Image J; US National Institutes of Health, Bethesda, MD; http://rsb.info.nih.gov/ij/, 1997-2005). Confocal image analysis was performed as previously described. ${ }^{27}$

For Western blot analysis of eGFP-NPM mutant A subcellular distribution, NIH-3T3 cells transfected with either pEGFP-C1 or pEGFP-C1$N P M \mathrm{~mA}$ were incubated with $20 \mathrm{ng} / \mathrm{mL}$ LMB (or vehicle, as control) in absence of cycloheximide for either 3 or 6 hours. Cells were then harvested, washed in phosphate-buffered saline (PBS) and lysed in hypotonic buffer according to the method of Schreiber et $\mathrm{al}^{28}$ slightly modified. The supernatant was saved as cytoplasmic fraction. The pellet fraction, containing nuclei, was washed with hypotonic buffer, solubilized in hypertonic buffer and boiled in SDS before loading. Equivalent dilutions (representing equal number of cells) of the cytoplasmic and pellet fractions were prepared for Western blot analysis of eGFP-NPM mutant A distribution with an anti-GFP monoclonal antibody (Roche, Indianapolis, IN).

The OCI/AML3 cell line cells were seeded in growth medium $\left(10^{6}\right.$ cells $/ \mathrm{mL}$ in 24 -well plates) and incubated at $37^{\circ} \mathrm{C}$ with $5 \% \mathrm{CO}_{2}$ for 5 hours. Following overnight incubation with LMB $(20 \mathrm{ng} / \mathrm{mL})$, cells were washed in PBS, centrifuged, fixed in B5, and embedded in paraffin for immunostaining. Primary leukemic cells from a NPMc ${ }^{+}$patient with $\mathrm{AML}$ with a high peripheral blood count were processed in the same way. 


\section{Immunostaining procedures}

For the Flag-HA constructs, H1299-fixed cells were permeabilized with $0.2 \%$ Triton-X 100 (10 minutes) and blocked with $10 \%$ antigoat serum (30 minutes). Primary antibody anti-HA (1:1000; Roche) was incubated with cells ( 1 hour), followed by secondary antibody Alexa 568-conjugated antirat (1:1000; Molecular Probes) for 30 minutes. Nuclei were visualized by DAPI staining. Images were collected using a Nikon Eclipse E400 microscope and a $40 \times / 1.3$ NA oil objective. Pictures were captured by the Spot Camera coupled with Spot 4.09 imaging software (Diagnostic Instruments, Sterling Heights, MI).

The nuclei of NIH-3T3 cells transfected with pEGFP-C1-NPM mutants and $\mathrm{pEGFP}-\mathrm{C} 1-N P M$ wt were visualized with propidium iodide. $\mathrm{pEGFP-C1-}$ NPM mutants transfected NIH-3T3 were immunostained with a specific antinucleolin antibody followed by a secondary Texas-Red-conjugated goat antimouse antibody (Southern Biotechnology Associates, Birmingham, AL); nuclei were counterstained with TO-PRO-3 (Molecular Probes). Paraffin-embedded samples from the OCI/AML3 cell line and 1 patient with $\mathrm{NPMc}^{+}$AML were immunostained with anti-NPM monoclonal antibody. ${ }^{8,29}$ The primary antibody was revealed using the immunoalkaline phosphatase APAAP technique ${ }^{8,29}$ and hematoxylin counterstain or a secondary Alexa 488-conjugated goat antimouse antibody (Molecular Probes) and propidium iodide counterstain.

\section{Cellular extracts, Western blotting, and coprecipitation studies}

Nuclear and cytoplasmic extracts were prepared either using the NE-PER Nuclear and Cytoplasmic Extraction kit (PIERCE Biotechnology, Rockford, IL) or according to the Schreiber's method, ${ }^{28}$ slightly modified. For immunoprecipitation, cells were lysed in $1 \mathrm{~mL}$ ice-cold lysis buffer $(0.5 \%$ NP-40, $150 \mathrm{mM} \mathrm{NaCl}, 25 \mathrm{mM}$ Tris [pH 7.5], $1 \mathrm{mM}$ EDTA, $1 \mathrm{mM} \mathrm{Na}_{3} \mathrm{VO}_{4}$, $1 \mu \mathrm{g} / \mathrm{mL}$ leupeptin, $1 \mu \mathrm{g} / \mathrm{mL}$ aprotinin, and $1 \mathrm{mM}$ phenylmethylsulfonyl fluoride). After incubation on ice (20 minutes), lysates were centrifuged at $14000 \mathrm{~g}\left(10\right.$ minutes at $\left.4^{\circ} \mathrm{C}\right)$ and then incubated with $4 \mu \mathrm{g}$ of either control immunoglobulin G (IgG), rabbit anti-NPMm (Sil-C), or mouse anti-NPM (clone 376), ${ }^{8}$ followed by $30 \mu \mathrm{L}$ Protein A/G Plus-agarose beads (Santa Cruz Biotechnology, Santa Cruz, CA) rocking overnight at $4^{\circ} \mathrm{C}$. Beads were extensively washed with buffer containing $0.1 \%$ NP-40, $150 \mathrm{mM} \mathrm{NaCl}, 25$ $\mathrm{mM}$ Tris (pH 7.5), 1 mM EDTA, and inhibitors. Proteins were separated by SDS-polyacrylamide gel electrophoresis (SDS-PAGE), transferred to polyvinylidene difluoride (PVDF; Millipore, Billerica, MA), and probed with either rabbit polyclonal anti-Crm1 (Santa Cruz Biotechnology) or mouse monoclonal anti-Crm1 antibody (BD Transduction Laboratories, Palo Alto, CA), followed by horseradish peroxidase-conjugated secondary antibodies. Polypeptides were detected using the enhanced chemiluminescence (ECL) method (Amersham Bioscience, Uppsala, Sweden).

\section{Results}

\section{All NPM exon-12 mutations create a leucine-rich NES motif in the carboxy-terminal portion of the NPM protein}

Analysis of the 6 NPM mutant forms (A to F) originally identified in $\mathrm{NPMc}^{+} \mathrm{AML}(\text { Table } 1)^{8}$ revealed a putative nuclear export signal (NES) motif $^{26}$ in the C-terminal portion of NPM proteins, which was suggested to be responsible for abnormal NPM cytoplasmic expression. This hypothesis implies that a NES motif is present in all NPM mutant proteins generated by NPM exon-12 mutations.

Therefore, we searched for additional exon-12 NPM mutations in patients with AML. Table 1 lists the 29 NPM mutations that have been so far identified by mutational screening of a total of 1554 patients with AML. ${ }^{8,22-24,30-32}$ In addition to the 6 NPM mutants forms (A to F) we initially reported, ${ }^{8}$ they include 3 new mutations ( $G$ to $I$ ) that we identified in 107 pediatric patients, ${ }^{30} 6$ new mutations $\left(G_{m}\right.$ to $\left.Q_{m}\right)$ that we identified in 401 patients with AML with normal karyotype of the protocol 99 of the German AML Cooperative Group (AMLCG), ${ }^{23} 9$ new mutations (numbered in Table 1 as 1, 3, 4, 6, 7, 12, 13, 10, and 14) reported by Dohner et al, ${ }^{22} 4$ new mutations $\left(G^{\dagger}\right.$ to $\left.J^{\dagger}\right)$ reported by Suzuki et al, ${ }^{31}$ and 1 new mutation $\left(\mathrm{I}^{\delta}\right)$ reported by Verhaak et al. ${ }^{24}$

We analyzed the NPM mutant proteins derived from the 29 mutation variants listed in Table 1 for the presence of a NES motif, as defined by a short stretch of amino acids characterized by multiple hydrophobic residues with typical spacing (eg, $\psi \mathrm{XXX} \psi \mathrm{XX} \psi \mathrm{X}$, where $\psi$ indicates large hydrophobic residues such as leucine, isoleucine, methionine, valine, or phenylalanine). ${ }^{33}$

The most frequent NES motif was L-xxx-V-xx-V-x-L that was present in $20(69 \%)$ of 29 NPM leukemic mutants. Nine $(31 \%)$ of the 29 mutants carried a NES motif in which the valine at the second position of NES motif was replaced with another hydrophobic amino acid. In particular, 3 (10.4\%) of 29 mutants carried the L-xxx-L-xx-V-x-L motif; 2 (6.9\%) of 29 mutants (Table 1; mutations $\mathrm{G}$ and $\mathrm{H}$ ) displayed the L-xxx-F-xx-V-x-L motif; 2 (6.9\%) of 29 mutants (Table 1; mutations 10 and 14) had the L-xxx-C-xx-V-x-L motif; and 1 (3.4\%) of 29 mutants (Table 1; mutation 1) exhibited the L-xxx-M-xx-V-x-L motif. Of the 29 mutants, $1(3.4 \%)$ (Table 1; mutation 6$)$, generated by a deletion of 7 nucleotides and insertion of 14 nucleotides, showed at the C-terminus the sequence LWQDFLNRLFKKIV. Although this sequence slightly deviates from the accepted NES consensus L-X-(2,3)-(LIVFM)-x(2,3)-L-X-(LI) ${ }^{33}$ present in the other $29 \mathrm{mu}-$ tants, it is still consistent with a leucine-rich NES because of the presence of 5 hydrophobic residues (leucine and phenylalanine) within a region of 10 amino acids. ${ }^{34}$

In conclusion, generation of an additional NES motif in the C-terminal portion of the NPM protein is one common consequence of NPM exon-12 mutations in $\mathrm{NPMc}^{+} \mathrm{AML}$.

\section{Export of NPM leukemic mutants is NES dependent}

Finding an additional NES motif suggests cytoplasmic dislocation of NPM may be due to a more active transport of the NPM mutants by $\mathrm{Crm} 1$ (exportin 1), ${ }^{35}$ the receptor for the NES motif of yeast and mammalian proteins. ${ }^{36,37}$

To test this hypothesis, we looked for perturbations in the nucleo-cytoplasmic transport of NPM mutant proteins in the presence/absence of the specific Crm1 inhibitor LMB. ${ }^{35} \mathrm{H} 1299$ or NIH3T3 cells were transfected with plasmids expressing NPM mutants A to D, fused to enhanced green fluorescent protein (eGFP), or NPM wild-type as control, and analyzed by immunofluorescence before and after treatment with LMB. In basal conditions, all 4 NPM mutant proteins were localized in the cytoplasm while NPM wild-type was restricted to the nucleoli, as expected (Figure 1A). However, LMB treatment completely relocated the cytoplasmic NPM mutants A to D into the nucleus (Figure 1B). Identical results (not shown) were obtained in NIH-3T3 cells transfected with mutant $\mathrm{A}$ and treated with ratjadones $\mathrm{A}$ and $\mathrm{C}$ that inhibit specifically the $\mathrm{Crm} 1^{38}$ with the same mechanism as LMB. ${ }^{39}$ Time course experiments with mutant A showed that about $50 \%$ of protein was relocated in the nucleus at 20 minutes and the process was completed after 1 hour (Figure 1C-D). Western blot analysis of eGFP-NPM mutant A subcellular distribution in LMB-treated NIH-3T3 cells confirmed a time-dependent accumulation of the intact eGFP-NPM mutant A protein $(64 \mathrm{kDa})$ in the pellet fractions (containing the nuclei) (Figure 1E). No alteration of eGFP protein 
Table 1. Changes in tryptophans 288 and 290 and creation of a NES motif in NPM mutant proteins generated by 29 NPM exon-12 mutations in 1554 patients with AML

\begin{tabular}{|c|c|c|c|c|c|c|}
\hline Mutation & & & Nucleotide sequence & & & Protein sequence \\
\hline $\mathrm{WT}^{*}$ & gaccaagaggctattcaagatct & ctg & gcag & t & ggaggaagtctctttaagaaaatag & 286-DLWQWRKSL \\
\hline A & gaccaagaggctattcaagatct & ctgTCTG & gcag & t & ggaggaagtctctttaagaaaatag & 286-DLCLAVEEVSLRK \\
\hline B & gaccaagaggctattcaagatct & ctgCATG & gcag & t & ggaggaagtctctttaagaaaatag & 286 -DLCMAVEEVELLRK \\
\hline $\mathrm{C}$ & gaccaagaggctattcaagatct & ctgCGTG & gcag & t & ggaggaagtctctttaagaaaatag & 286-DLCVAVEEEVSLRK \\
\hline D & gaccaagaggctattcaagatct & ctgCCTG & gcag & t & ggaggaagtctctttaagaaaatag & 286 -DLCLAVEEEVSLRK \\
\hline$E$ & gaccaagaggctattcaagatct & $\operatorname{ctg}$ & gcag & t CTCTTGCCC & Caagtctctttaagaaaatag & $286-\mathrm{DL} W Q S \underline{S} \mathrm{AQV}$ SLRK \\
\hline $\mathrm{F}$ & gaccaagaggctattcaagatct & $\operatorname{ctg}$ & gcag & t CCCTGGAGP & Aaagtctctttaagaaaatag & $286-\mathrm{DL} W Q S \underline{\text { LEKVSLLRK }}$ \\
\hline $\mathrm{E} \bullet$ & gaccaagaggctattcaagatct & $\operatorname{ctg}$ & gcag & t CCCTCGCCO & Caagtctctttaagaaaatag & 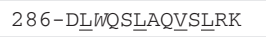 \\
\hline G・ & gaccaagaggctattcaagatct & $\operatorname{ctg}$ & gcag & t GCTTCGCCC & Caagtctctttaagaaaatag & $286-\mathrm{D} \underline{W Q C E}$ EAQVSSLRK \\
\hline $\mathrm{H} \bullet$ & gaccaagaggctattcaagatct & $\operatorname{ctg}$ & gcag & t GTTTTTCAA & Aaagtctctttaagaaaatag & $286-\mathrm{DL} W Q C \underline{F} S K \underline{V} S \underline{L} R K$ \\
\hline $\mathrm{G}_{\mathrm{m}}$ & gaccaagaggctattcaagatct & ctgCAGG & gcag & t & ggaggaagtctctttaagaaaatag & 286 -DLCRAVEEEVSLRK \\
\hline $\mathrm{K}_{\mathrm{m}}$ & gaccaagaggctattcaagatct & ctgCCGG & gcag & $t$ & ggaggaagtctctttaagaaaatag & 286 -DLCRAVEEEVSLRK \\
\hline $\mathrm{L}_{\mathrm{m}}$ & gaccaagaggctattcaagatct & ctgCCGCGG & Gag & $t$ & ggaggaagtctctttaagaaaatag & 286 -DLCRGVEEEVSLRK \\
\hline $\mathrm{N}_{\mathrm{m}}$ & gaccaagaggctattcaagatct & $\operatorname{ctgCCAG}$ & gcag & t & ggaggaagtctctttaagaaaatag & $286-\mathrm{DLCQA} \underline{\mathrm{V} E E \underline{V} S \mathrm{~L} R K}$ \\
\hline $\mathrm{O}_{\mathrm{m}}$ & gaccaagaggctattcaagatct & ctgTTTG & gcag & $t$ & ggaggaagtctctttaagaaaatag & 286-DLCLAVEEEVSSLRK \\
\hline$Q_{m}$ & gaccaagaggctattcaagatct & ctgTCGG & gcag & t & ggaggaagtctctttaagaaaatag & 286-DLCRAVEEEVSLRK \\
\hline 1 & gaccaagaggctattcaagatct & $\operatorname{ctg}$ & gcagTCCA & t & ggaggaagtctctttaagaaaatag & 286-DLWQSMEEVSLRK \\
\hline 3 & gaccaagaggctattcaagatct & ctgTCAT & gcag & t & ggaggaagtctctttaagaaaatag & 286-DLCHAVEEESLLRK \\
\hline 4 & gaccaagaggctattcaagatct & ctgCTTG & gcag & $t$ & ggaggaagtctctttaagaaaatag & 286 -DLCLAVEEEVSLRK \\
\hline 6 & gaccaagaggctattcaagatct & $\operatorname{ctg}$ & gса AGATTTCTTAAATC & & gtctctttaagaaaatag & 286-DLWQDFLNRLFKKIV \\
\hline 7 & gaccaagaggctattcaagatctATGC & $\operatorname{ctg}$ & gcag & $t$ & ggaggaagtctctttaagaaaatag & 286-DLCLAVEEEVSSLRK \\
\hline 12 & gaccaagaggctattcaagatct & ctgGCCC & gcag & t & ggaggaagtctctttaagaaaatag & 286-DLCAAEEEVSLRK \\
\hline 13 & gaccaagaggctattcaagatct & ctgTAAG & gcag & t & ggaggaagtctctttaagaaaatag & 286 -DLCKAVEEEVSLRK \\
\hline 10 & gaccaagaggctattcaagatct & $\operatorname{ctg}$ & gcag & $\operatorname{tgCTGCTCCC~}$ & aagtctctttaagaaaatag & $286-\mathrm{D} \_W Q C \underline{C} S Q \underline{Q}$ SLRK \\
\hline 14 & gaccaagaggctattcaagatct & $\operatorname{ctg}$ & gcag & $t$ TATTTTCC & Caagtctctttaagaaaatag & $286-\mathrm{D} \underline{W} W \mathrm{QC} \underline{\mathrm{C}} \mathrm{SQ} \underline{\mathrm{V}} \underline{\mathrm{LRK}}$ \\
\hline $\mathrm{G}^{\dagger}$ & gaccaagaggctattcaagatct & ctgTTTG & gcag & t & ggaggaagtctctttaagaaaatag & 286 -DLCLAVEEEVELRK \\
\hline $\mathrm{H}^{\dagger}$ & gaccaagaggctattcaagatct & ctgCTTG & gcag & $t$ & ggaggaagtctctttaagaaaatag & 286-DLCLAVEEEVSSLRK \\
\hline$I^{\dagger}$ & gaccaagaggctattcaagatct & ctgTAAG & gcag & t & ggaggaagtctctttaagaaaatag & 286 -DLCKAㅌEEVSLLRK \\
\hline $\mathrm{J}^{\dagger}$ & gaccaagaggctattcaagatct & ctgTATG & gcag & t & ggaggaagtctctttaagaaaatag & 286 -DLCMAVEEEVSLRK \\
\hline $\mathrm{I}^{\triangle}$ & gaccaagaggctattcaagatct & ctgCAGA & gcag & t & ggaggaagtctctttaagaaaatag & 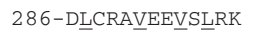 \\
\hline
\end{tabular}

Mutations $A$ to $F$ refer to those originally identified in Falini et al. ${ }^{8}$ Mutations from $\mathrm{E} \bullet$ to $\mathrm{H} \bullet$ refer to those identified in childhood $A M L$ cases in Cazzaniga et al. ${ }^{30}$ Mutations $\mathrm{G}_{\mathrm{m}}$ to $Q_{m}$ refer to those identified in Schnittger et $\mathrm{al}^{23}$ in protocol 99 of the German $A M L$ cooperative group; mutations $1,3,4,6,7,12,13,10$ and 14 according to Döhner et al 22 ; mutations $\mathrm{G}^{\dagger}, \mathrm{I}^{\dagger}, \mathrm{H}^{\dagger}$ and $\mathrm{J}^{\dagger}$ according to Suzuki et al ${ }^{31}$; mutation $\mathrm{I}^{\Delta}$ according to Verhaak et al. ${ }^{24}$ Lowercase letters in nucleotide sequences indicate wild-type sequence residues; capital letters, mutated nucleotides. Underlined text indicates leucine-rich NES motif; italics, tryptophan (W) residues.

WT indicates wild type; L, leucin; V, valine; F, phenylalanine; $M$, methionine; C, cysteine.

*NM_002520.

(27 kDa) localization was observed in LMB-treated cells (only a mild contamination of the pellet fractions by the overexpressed protein was detected). In untreated cells, either eGFP or eGFPNPM mutant A protein were found (except for the contaminant part) only in cytoplasmic fractions, as expected.

Confocal microscopy analysis of eGFP-NPM mutant A (L-xxx$\mathrm{V}-\mathrm{xx}-\mathrm{V}-\mathrm{x}-\mathrm{L}$ NES motif) showed that relocalization of the mutated protein induced by LMB was limited to the nucleoplasm (Figure 2A, top panel). This localization clearly differed from that of eGFP-NPM wild-type protein which, as expected, was located in the nucleoli, whether LMB was present or not (Figure 2A, middle panel). Transient transfection with pEGFP-C1-NPMmA and immunostaining for nucleolin/C23 followed by 3D reconstruction confirmed that intranuclear location of NPM mutant A (nucleoplasm, green) and nucleolin (nucleoli, red) were mutually exclusive (Figure 2A, lower panel). A nucleoplasmic staining pattern (not shown) was observed after LMB treatment with 1 rare NPM mutant bearing the NES motif L-xxx-F-xx-V-x-L (mutant G; Table 1).

LMB also caused nuclear relocation of the mutant NPM in the OCI/AML3 cells (Figure 2B) that are known to carry the exon-12 mutation A. ${ }^{27}$ In these cells, Crm1 and NPM mutant A were shown to coprecipitate, indicating a physical interaction between the 2 molecules (Figure 2C). Leptomycin induced similar nuclear relocation of NPM in primary leukemic cells of 1 patient with $\mathrm{NPMc}^{+}$ AML (not shown).
Finally, the requirement of a NES motif for abnormal cytoplasmic expression of NPM was further documented by testing the subcellular localization of a modified eGFP-NPM mutant A construct where the 2 valine residues in the NES motif were replaced by glycines. Transient transfection of NIH-3T3 cells with this construct showed that the NPM mutant accumulated in the nucleoplasm, whether LMB was present or not (Figure 3, right panel).

These results provide evidence that aberrant cytoplasmic localization of NPM mutants in $\mathrm{NPMc}^{+} \mathrm{AML}$ is a NES-dependent event.

\section{Two NESs are better than one}

The NPM wild-type protein contains a putative physiologic N-terminus NES motif at position $92-104 .{ }^{40}$ To assess the contribution of the physiologic versus additional C-terminus NES motif created by the mutations in causing abnormal cytoplasmic expression of NPM, we tested the subcellular localization of modified eGFP-NPM mutant A constructs where the second and third hydrophobic residues of the N-terminal or C-terminal NES motif were replaced by glycines (Figure 3). Transient transfection of NIH-3T3 with such constructs showed that the NPM mutants lacking either the physiologic (N-terminus) or the new mutationrelated (C-terminus) NES accumulated in the nucleoplasm (Figure 3 , middle and right panels), indicating that the nuclear export and 
A

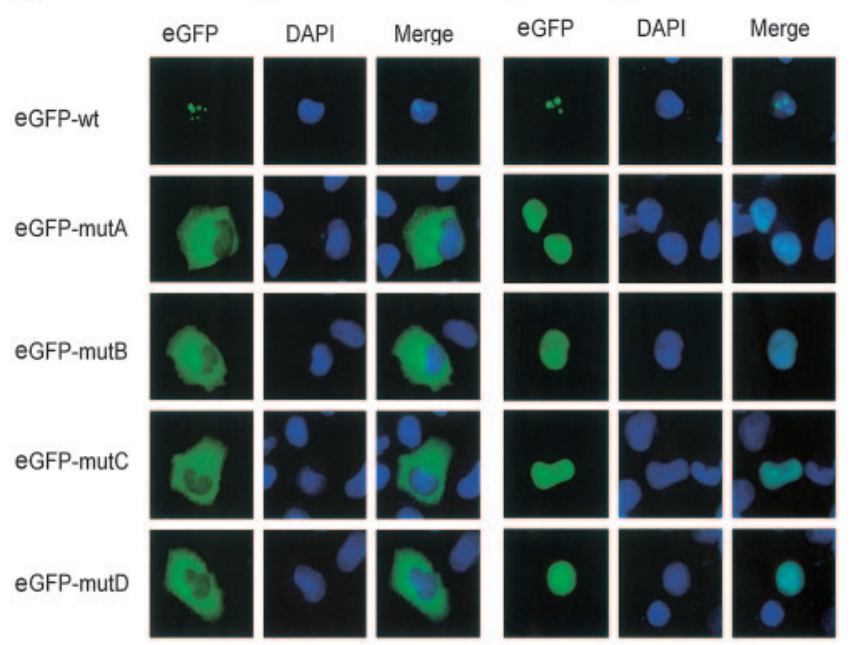

C

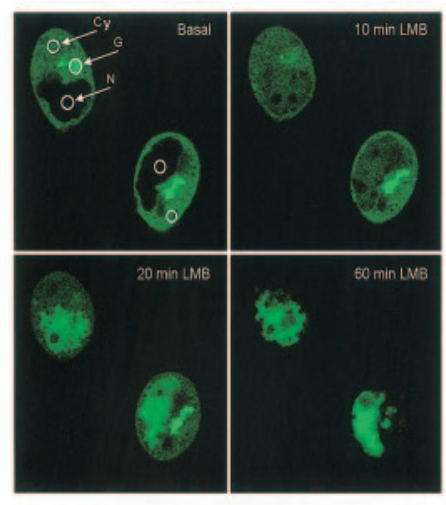

E
D

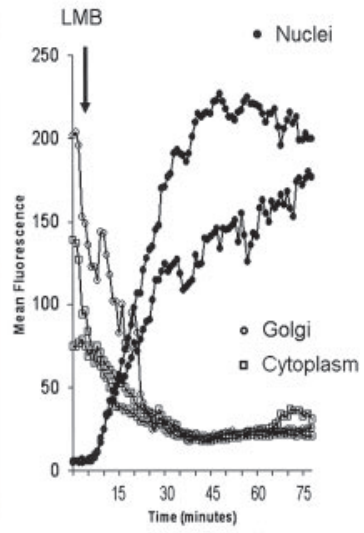

$6 \mathrm{~h}$

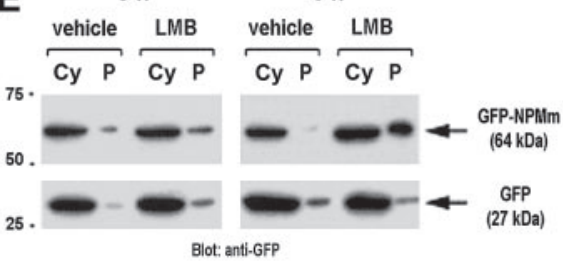

50

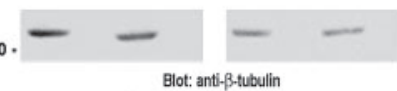

Figure 1. Nuclear export of NPM mutants is NES dependent. (A-B) H1299 cells transfected with cDNA encoding for the NPMwt and the NPM mutant proteins $A$ to $D^{8}$ in the absence (A) and presence (B) of LMB. eGFP-NPMwt localizes in the nucleoli both in the presence and absence of LMB. In the absence of LMB, all GFP mutants (A-D) show the expected aberrant cytoplasmic location (A). The same eGFP-mutants are completely relocated in the nucleus in the presence of $L M B(B)$. (C-E) Time-course analysis of $L M B$ induced nuclear retention of eGFP-NPMmutA in NIH-3T3 cells. (C) Confocal microscope images of NIH-3T3 cells at the indicated time points. $\bigcirc$ represents the regions (regions of interest [ROIs]) where the mean fluorescence emission of eGFP-NPMmutA was calculated in the experiment (eg, nucleus [N], cytoplasm [Cy], and the Golgi apparatus [G]). (D) Time-course measurements of mean fluorescence in the selected areas (ROIs) of NIH-3T3 cells. LMB addition produced a loss of fluorescence in the cytoplasm and Golg apparatus and a concomitant increase in the mean fluorescence of the nucleoplasm. ImageJ software was used for the generation of ROls and time-course analysis. (E) Western blot analysis of eGFP-NPMmutA subcellular distribution in NIH-3T3 cells with anti-GFP monoclonal antibody. LMB treatment induces a time-dependent accumulation of eGFP-NPMmutA in the pellet fractions $(P)$. Purity of the subcellular fractions was assessed by stripping the membrane and reblotting with an anti- $\beta$-tubulin antibody (bottom panel). Only a nonsignificant contamination was detected for the overexpressed proteins (as evident in the GFP blotting) (middle panel). In untreated cells, eGFP-NPMmutA (except for the contaminant part) was found only in the cytoplasmic fractions (Cy). The experiment was conducted in absence of cycloheximide so that continuous presence of GFP-NPMmA in the cytoplasmic fraction during LMB treatment was detected with time.

cytoplasmic accumulation of NPM mutants can only occur in the presence of 2 NES motifs (Figure 3, left panel); LMB had no effect on subcellular localization of the 2 mutants (not shown).

\section{Role of tryptophans 288 and 290 in nucleolar binding and nuclear export of NPM leukemic mutants}

After LMB treatment, NPM mutant A relocated into the nucleoplasm but not in the nucleoli (Figure 2A, top and bottom panels). This finding was not unexpected as both tryptophans 288 and 290 are mutated in this sequence, and the homologous residues in rats (positions 286 and 288) appear to be critical for nucleolar localization of wild-type NPM, with mutations of both tryptophans resulting in nucleoplasmic delocalization of the protein. ${ }^{41}$

All 29 NPM mutant proteins carried a mutated tryptophan 290, while tryptophan 288 was retained in $9(31 \%)$ of 29 mutants (Table 1). Analysis of tryptophan mutations and NES motif distribution in NPM mutants revealed that the NES most commonly found in the NPM mutants (ie, L-xxx-V-xx-V-x-L) is always associated with mutations of both tryptophans, while tryptophan 288 is retained only in the NPM mutants carrying variants of the most common NES motif (ie, substitution of the valine at the second position of NES with leucine, phenylalanine, cysteine, or methionine).

We used constructs expressing NPM mutant E (Table 1) fused to eGFP to assess the effect of the single mutation at tryptophan 290 on nucleolar binding. After treatment with LMB, cytoplasmic mutant $\mathrm{E}$, like mutant A, relocated to the nucleoplasm; however, mutant $\mathrm{E}$ also retained some capability to target nucleoli, as traces of GFP were detected in these structures (Figure 4). A similar pattern was observed using a derivative construct of mutant A with tryptophan 290 reinserted by site-directed mutagenesis (A290W; Figure 4). Notably, when both tryptophans were reinserted in mutant A (C288W + A290W), the mutant protein localized completely to the nucleoli whether LMB was present or not (Figure 4), suggesting a dose effect for tryptophan molecules.

These findings provide evidence that: (1) tryptophan 290 is mutated in all NPM leukemic mutants; (2) tryptophan 288 is retained only in NPM mutants carrying a NES variant other than the most common motif L-xxx-V-xx-V-x-L; (3) as in the rat, ${ }^{41}$ tryptophans 288 and 290 are both important for nucleoli targeting; (4) maximum dislocation of NPM mutants from nucleoli to nucleoplasm occurs when both tryptophans are mutated; and (5) NES-dependent nuclear export of the NPM mutant A is blocked following reinsertion of both tryptophans 288 and 290.

\section{Identification of the mutant NPM protein by a novel specific antibody}

Currently used anti-NPM antibodies ${ }^{17}$ do not discriminate between wild-type and mutated NPM proteins, which, since NPM exon-12 mutations are heterozygous, ${ }^{8}$ are both found in leukemic cells. 
Figure 2. LMB relocates NPM mutants in the nucleoplasm. (A) Confocal microscopy of NIH-3T3 cells transfected with pEGFP-C1-NPMmA or pEGFP-C1-NPMwt in presence and absence of $L M B$. In basal conditions, the mutant $A$ is cytoplasmic (top left) but relocates in the nucleoplasm (top right) following incubation with LMB. eGFP-NPMwt localizes in the nucleoli both in the presence and absence of LMB (middle panel, left and right). The bottom panel shows LMB-treated cells transfected with pEGFP-C1-NPMmA (green) and immunostained with an antinucleolin (C23) monoclonal antibody (Texas red)-labeling nucleoli (arrow). The nuclear membrane is blue (TO-PRO-3 staining). After LMB, the eGFP-NPM mutant $A$ was relocated in the nucleoplasm. Nucleolar exclusion was confirmed by an electronic cut of the nucleolar surface (inset in the bottom right figure). (B) The anti-NPM antibody 376 labels both nucleoli and cytoplasm of OCl/AML3 cells in the absence of LMB (left). Incubation with LMB results in nucleoplasmic relocation of the cytoplasmic NPM (right). Images were collected as described $^{27}$ using a Zeiss Plan Apochromat $100 \times / 1.4$ NA oil objective. (C) OCI-AML3 and U937 cell lysates were subjected to immunoprecipitation (IP) with either control IgG or rabbit anti-NPMm (Sil-C) and mouse anti-NPM (Cl. 376): Western blots with anti-Crm1 antibodies are shown in the top panels. Blotting with the antiNPMm (Sil-C) and anti-NPM (Cl. 376) antibody are also shown (middle and bottom panels). WCL indicates $\mathrm{OCl}$ AML3 whole-cell lysate included as control.
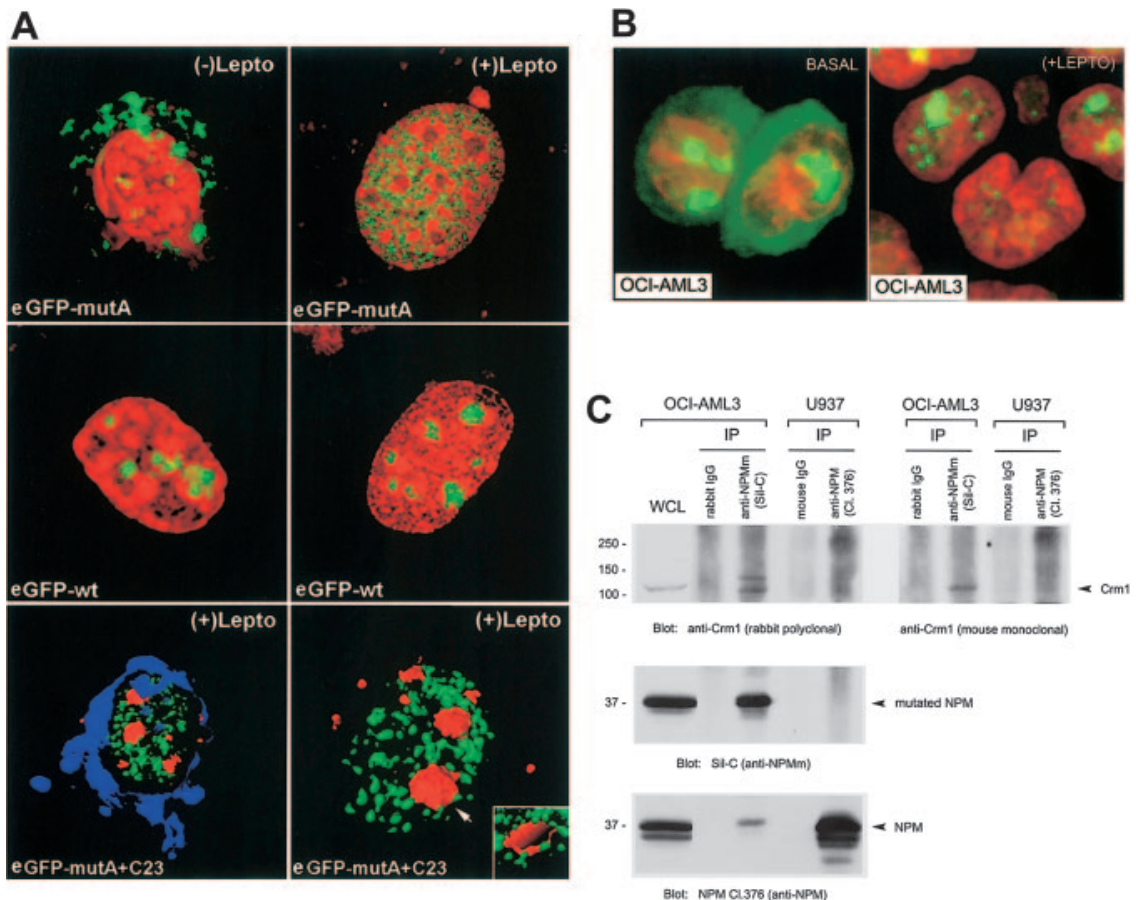

Although the NPM mutant constructs localize exclusively in the cytoplasm in transfected cells, ${ }^{8}$ subcellular distribution (nuclear vs cytoplasmic) of NPM mutant proteins in primary $\mathrm{NPMc}^{+} \mathrm{AML}$ samples could not be investigated because of interference of NPM wild-type protein. To address this issue, we developed a polyclonal antibody (Sil-C) specifically directed against NPM mutants. Upon Western blotting, Sil-C recognized a specific 37-kDa band only in whole-cell lysates from $\mathrm{NPMc}^{+} \mathrm{AML}$ primary cells (Figure 5A, top panel; patients 1-3) and not in NPMc- AML samples (patients 4 and 5). Conversely, the anti-NPM monoclonal antibody 376, which also reacts with wild-type NPM, detected the $37-\mathrm{kDa}$ band in all 5 patients (Figure 5A, bottom panel). In patient 2 (carrying NPM mutation A), the Sil-C antibody recognized the 37-kDa band only in the cytoplasm (Figure 5B, top panel) while the 376 antibody detected it in both nuclear and cytoplasmic fractions, as expected (Figure 5B, bottom panel).

These findings were confirmed by immunohistochemical staining of bone marrow biopsies from 10 patients with $\mathrm{NPMc}^{+} \mathrm{AML}$
(Figure 5C). Interestingly, the Sil-C antibody not only stained the 7 $\mathrm{NPMc}^{+}$AMLs carrying the mutation A, but also those carrying the mutation B (1 case) and D (2 cases). In all samples, Sil-C gave cytoplasmic-restricted positivity (Figure 5D), which, in competition experiments, was antagonized by the peptide used as immunogen (not shown). The same peptide did not antagonize cytoplasmic reactivity of a monoclonal antibody (clone 376) directed against a different epitope of NPM (not shown). As expected, immunostaining with monoclonal anti-NPM antibodies recognizing wild-type and mutated NPM ${ }^{17}$ resulted in nuclear plus cytoplasmic positivity of the leukemic cells (Figure 5E). The Sil-C antibody did not stain leukemic cells in any of the 10 AMLs with an unmutated NPM gene and nucleus-restricted expression of wild-type NPM $\left(\mathrm{NPMc}^{-}\right)$ used as a negative control (Figure 5F-G).

These findings confirm that the endogenous mutated NPM proteins localize exclusively in the cytoplasm of primary $\mathrm{NPMc}^{+}$ AML cells, suggesting that export of mutated NPM protein from the nucleus is highly efficient and more rapid than nuclear import.

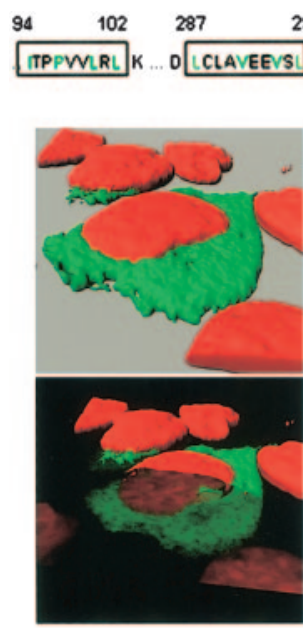

NPM-mutA
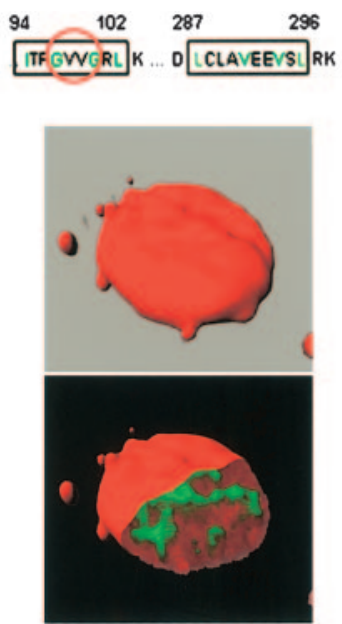

NPM-mutA_NO_1st NES
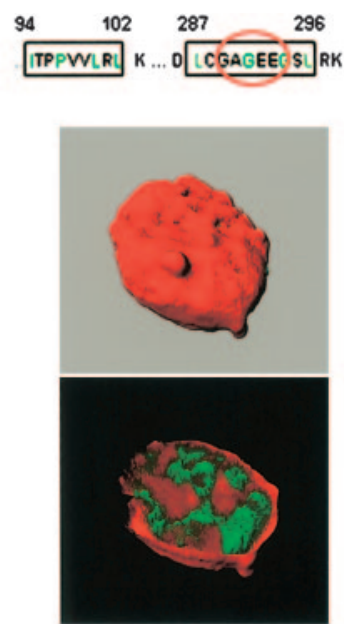

Figure 3. Export of NPM mutants require 2 NES motifs. Confocal microscope analysis of $\mathrm{NIH}-3 \mathrm{~T} 3$ transfected with either pEGFP-C1-NPMmA (left panel), pEGFP. C1-NPM_MUT_A_NO_1st_NES (middle panel), or pEGFP-C1-NPM_MUT_A_NO_2nd_NES (right panel). NPM mutant A containing both NES motifs localizes in the cytoplasm. Disruption of either $\mathrm{N}$-terminal (middle panel) or C-terminal (right panel) NES motif in the NPM mutant A impedes nuclear export of the protein that appears localized in the nucleoplasm (middle and right panels, bottom). Images were collected as described ${ }^{27}$ using a

NPM-mutA_NO_2nd NES Zeiss Plan Apochromat $100 \times / 1.4$ NA oil objective. 
(-) LEPTO
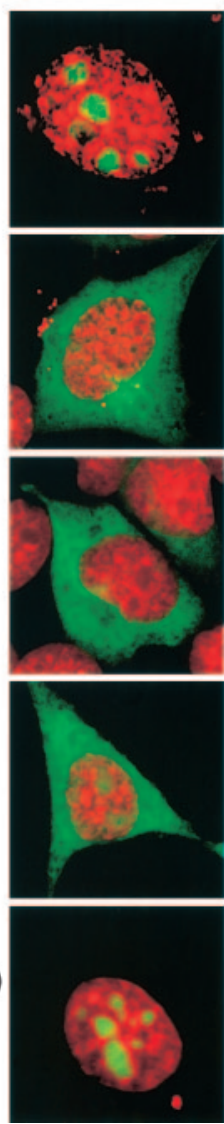

NPM mut E

DLWQSLAQVSLRK

NPM mut A (A290W)

DLCLWVEEVSLRK

NPM mut $A(C 288 W+A 290 W)$

DLWLWVEEVSLRK
(+) LEPTO

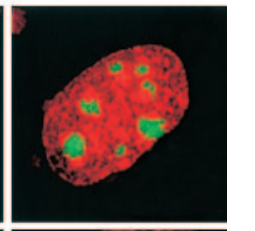

\section{Discussion}

The present study shows how specific mutations on the NPM exon-12 sequences severely impair NPM nucleo-cytoplasmic trafficking in transfected cells, AML cell line OCI/AML3 (carrying exon-12 mutation A), and primary $\mathrm{NPMc}^{+} \mathrm{AML}$ cells. Consequent to the mutations, 2 major alterations occur at the C-terminus of NPM leukemic mutants: (1) generation of a leucine-rich NES motif that reinforces the Crm1-dependent nuclear export of mutated NPM proteins; and (2) loss of the C-terminus tryptophan(s) residues that are normally required for NPM binding to the nucleoli.

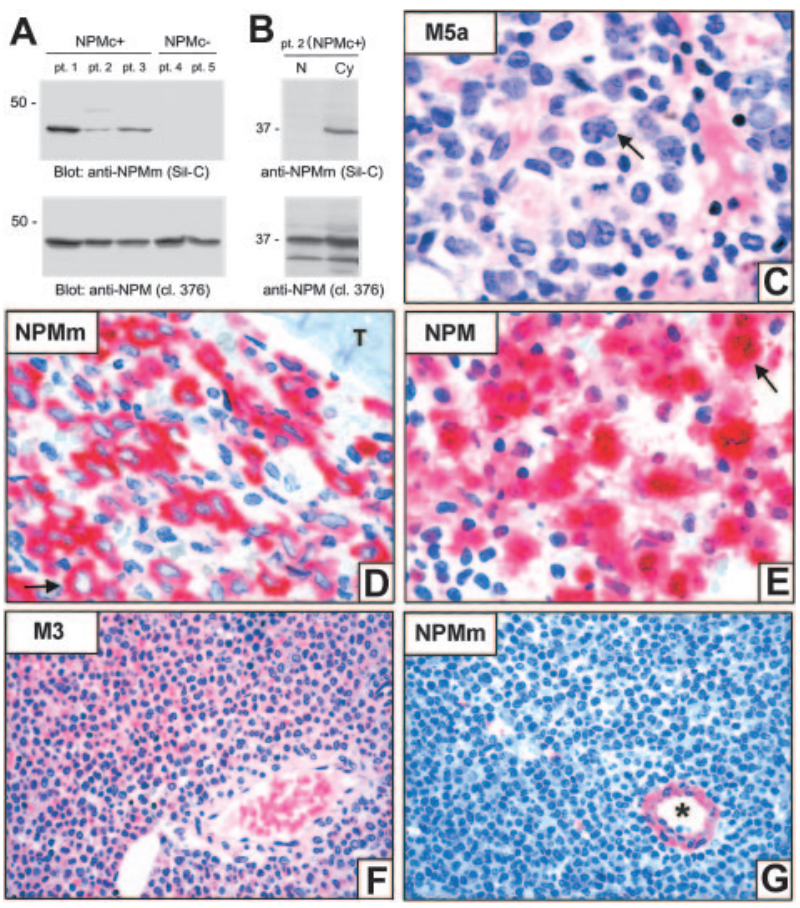

Figure 5. NPM mutants localize exclusively in the cytoplasm of leukemic cells. (A-B) Western blot analysis (Sil-C vs $376 \mathrm{mAb}$ ) of whole-cell lysates from either $\mathrm{NPMc}^{+}$or NPMc ${ }^{-}$AML cells $(\mathrm{A})$ and of nuclear $(\mathrm{N})$ and cytoplasmic (Cy) cell fractions from a patient with $A M L$ bearing NPM mutation $A(B)$. Sil-C recognizes specifically the mutated NPM protein in the cytoplasmic fraction of $\mathrm{NPMc}^{+}$primary leukemic cells. Results are representative of at least 3 independent experiments. (C-E) Bone marrow biopsy from a NPMc ${ }^{+}$AML bearing mutation A. (C) Typical appearance of FAB-M5a type AML; the arrow points to a large leukemic cell with kidney-shaped nucleus. (D) The Sil-C antibody that recognizes specifically the NPM mutant (NPMm) shows a cytoplasmic-restricted positivity of leukemic cells (arrow); T indicates a bone trabecula. (E) Monoclonal anti-NPM 376 that recognizes both the wild-type and mutant NPM proteins labels leukemic cells both in the cytoplasm and the nucleus (arrow). (F-G) Bone marrow biopsy from a case of promyelocytic leukemia lacking NPM mutations $\left(\mathrm{NPMc}^{-}\right.$). (F) Typical appearance of AML FAB-M3 (hematoxylineosin). (G) The Sil-C antibody does not stain leukemic cells (devoid of mutated NPM protein) and only cross-reacts with a vessel wall; the asterisk indicates the lumen of the vessel (APAAP technique; hematoxylin counterstain). Images were collected using an Olympus BX51 microscope and a UPlanFI $100 \times / 1.3$ NA oil (C-E) or a UPlanApo $40 \times / 0.85$ (F-G) objective. 

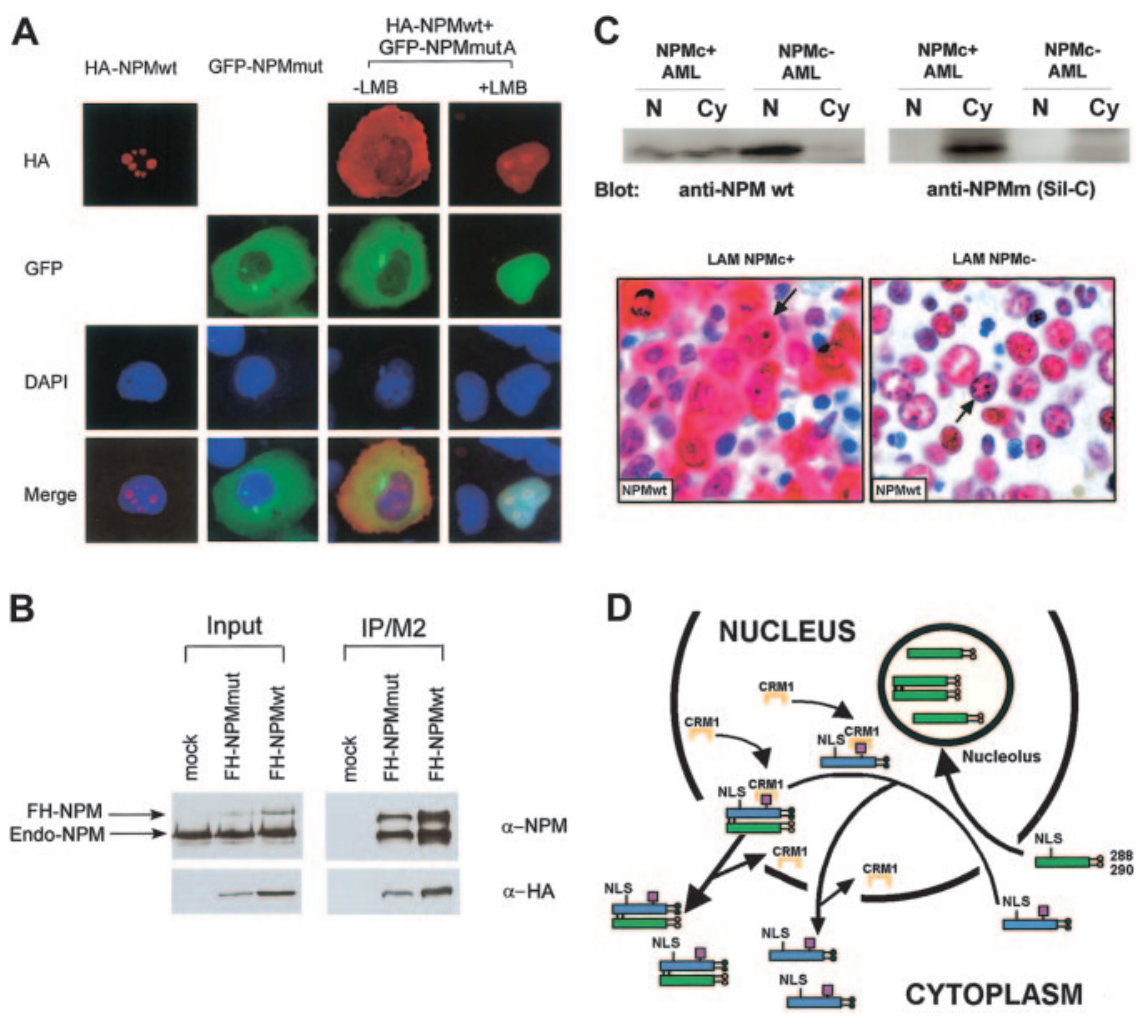

Figure 6. NPM mutant A acts as a dominant-negative on wild-type NPM. (A) Cotransfection of H1299 cells with pEGFP-C1-NPMmA and HA-NPM wild-type. About $30 \%$ of cells were transfected and in about $70 \%$ of transfected cells the NPM mutant causes partial recruitment of the wild-type NPM from nucleoli to the nucleoplasm and the cytoplasm. (B) Both FH-NPMwt and FH-NPM mutant A interact with the endogenous NPM in H1299 cells. FH-NPM wt and FH-NPM mutant A plasmids were stably transfected into $\mathrm{H} 1299$ cells. In the left panel, $5 \%$ of the whole-cell lysates from stably transfected FH-NPMwt, FH-NPM mutant A, or control H1299 cells were subjected to Western blot with either $\alpha$-NPM or $\alpha$-HA. In the right panel, the rest $95 \%$ of whole cell lysates were immunoprecipitated with $\alpha$-flag (M2), followed by Western blot with either $\alpha$-NPM or $\alpha$-HA. (C) Endogenous wild-type NPM protein is dislocated in the cytoplasm of NPMc ${ }^{+} \mathrm{AML}$ primary samples. In the top panel, nuclear and cytoplasmic extracts from either $\mathrm{NPMc}^{+}$or NPMc $^{-}$AML primary patient cells were subjected to Western blot analysis with either the anti-NPM mouse monoclonal antibody (clone FC-61991) from Invitrogen (anti-NPM wt) or the Sil-C antibody (anti-NPMm). A strong signal for NPMwt was evident in both the nuclear and cytoplasmic fraction of AML cells carrying NPM mutation but only in the nuclear fraction of NPMc ${ }^{-}$AML cells. The lower panel shows immunohistochemical study of 2 different AML samples with the anti-NPMwt antibody. The NPMc ${ }^{+}$AML sample expresses the wild-type NPM both in the nucleus and cytoplasm (arrow). In contrast, the NPMc- AML sample shows the expected nucleus-restricted expression of wild-type NPM (arrow). Images were collected using an Olympus BX61 microscope and a UPlanFI $100 \times / 1.3$ NA objective. (D) Hypothetical mechanism of altered nucleo-cytoplasmic traffic of NPM mutant A and wild-type NPM. Red circles indicate tryptophan residues; black circles, mutated tryptophans; magenta boxes, NES motif; blue boxes, mutated NPM protein; and green boxes, wild-type NPM protein. To simplify, the scheme shows only the binding of Crm1 to the mutant NES but not to the physiologic one, nor to the full ternary complex (Crm1-NES-ranGTP). NLS indicates nuclear localization signal.

The NPM wild-type protein contains a putative hydrophobic leucine-rich NES motif (I-xx-P-xx-L-x-L) within residues 94-102 which is conserved from Xenopus laevis to humans. ${ }^{40}$ Despite the presence of this motif, the NPM wild-type protein is mainly localized in the nucleoli suggesting that, in physiologic conditions, protein import greatly predominates over nuclear export. Interestingly, $\mathrm{rat}^{41}$ and human ${ }^{40}$ NPM wild-type protein mutants lacking the 2 tryptophans 288 and 290 delocalize in the nucleoplasm but not in the cytoplasm, ${ }^{40,41}$ strongly suggesting that the additional NES motif reinforces the leukemic NPM mutant ability to be exported from the nucleus to the cytoplasm. As the NES motif at the C-terminus of leukemic NPM mutant A is absent in the NPM wild-type protein, enhanced export could be due to the additive effects of the 2 NES and/or to an increased affinity of the new NES motif for Crm1. The results presented in this paper indicates that cytoplasmic accumulation of NPM mutants depends on the additive effect of the newly generated C-terminal NES motif.

The tryptophans 288 and 290 both appear to be important for nucleolar localization, with tryptophan 290 being perhaps more critical since it is mutated in all NPM mutants so far identified. Maximal inhibition of nucleolar binding and nucleoplasmic delocalization of mutants is observed when both tryptophans are mutated. Notably, mutations affecting both tryptophans 288 and 290 were detected in $20(69 \%)$ of 29 NPM mutants identified to date, and account for about $95 \%$ of all tryptophan mutations found in NPM leukemic mutants.

The correlation between the type of NES motif and mutations of tryptophans 288 and 290 is intriguing. The observation that the NES motif most commonly found in the NPM mutants (ie, $\mathrm{L}-\mathrm{x} x \mathrm{x}-\mathrm{V}-\mathrm{xx}-\mathrm{V}-\mathrm{x}-\mathrm{L}$ ) is always associated with mutations of both tryptophans and that tryptophan 288 is retained only in the NPM mutants carrying variants of the most common NES motif (ie, substitution of the valine at the second position of NES with leucine, phenylalanine, cysteine or methionine), suggest a functional difference between the NES motifs.

Taken together, our data clearly show that, for cytoplasmic accumulation of NPM to occur, the 2 NES motifs and tryptophan(s) mutations must act in concert. This view clearly differs from that of Nagakawa et al, ${ }^{26}$ who claimed the NES motif at the C-terminus was the only player in cytoplasmic dislocation of NPM leukemic mutants. The mechanism of altered nucleo-cytoplasmic traffic in $\mathrm{NPMc}^{+} \mathrm{AML}$ cells also emerges as different to what den Besten et $\mathrm{al}^{45}$ recently observed in transfected cells. In MT-Arf cells, leukemic NPM mutant A relocation after incubation with LMB is reported to be nucleolar, while we clearly demonstrate relocation of NPM mutant A by Crm1-inhibitors is nucleoplasmic in transfected 
NIH-3T3 cells. Several points need to be borne in mind when analyzing these discrepancies. Like Nakagawa et al, ${ }^{26}$ den Besten et $\mathrm{al}^{45}$ appear to disregard or underestimate the role of mutated tryptophan(s) 288 and 290 in the altered nucleo-cytoplasmic transport of NPM leukemic mutants. Artificial interference by the Arf protein in MT-Arf cells used by den Besten et $\mathrm{al}^{45}$ may be another factor. Finally, den Besten et $\mathrm{al}^{45}$ show that much wild-type NPM protein is delocalized in the cytoplasm by the NPM mutant A. Our immunohistologic studies of bone marrow biopsies using anti-NPM antibodies, including reagents specific for the wild-type and mutated NPM proteins, confirm the presence of cytoplasmicdislocated wild-type NPM in primary NPMc ${ }^{+} \mathrm{AML}$ cells, but also reveal that a residue of wild-type NPM remains in the nucleus (Figure 6C, bottom panels). Further studies are required to exactly quantify by fluorescence-based techniques the ratio of wild-type to mutated NPM in the subcellular compartments of $\mathrm{NPMc}^{+}$primary leukemic cells.

Based on these findings, the following scenario can be envisaged. Mutated NPM proteins that retain the 2 nuclear localization signals (NLSs) enter the nucleus. Their capability to bind nucleoli is hindered completely (when both tryptophans are mutated) or partially (when only tryptophan 290 is altered). Nucleoplasmic NPM mutants may become easily available for binding to Crm1 roaming in the nucleoplasm ${ }^{46}$ through the NES motifs. NPM mutants accumulate in the cytoplasm most likely because the NES-Crm1-mediated nuclear export of mutants is more efficient than nuclear import. The NPM leukemic mutants cause in turn partial delocalization of the NPM wild-type protein from the nucleus to the cytoplasm.

Our demonstration that antibodies can be produced to react with mutant, but not with wild-type, NPM proteins is of biological interest and potential clinical relevance. It might be feasible in the future to use intracellular antibodies ("intrabodies") 47 against NPM mutants to ablate protein function as an alternative to gene-based knockout technologies which do not appear to discriminate between wild-type and mutated NPM encoding RNAs (B.F. and A.L., unpublished observations, June 2005). The OCI/AML3 cell line may well serve as a preclinical model for screening intrabodies and other compounds designed to interfere with the NPM-altered nucleo-cytoplasmic traffic.
The NPM wild-type protein plays a critical role in the control of hematopoiesis (especially erythropoiesis) during embryonic development, ${ }^{15}$ and is involved in regulation of the Arf-p53 pathway ${ }^{9,11-14}$ and centrosome duplicaton. ${ }^{10,40}$ Our finding that in transfected cells NPM mutants recruit wild-type NPM to the nucleoplasm and cytoplasm suggests they interfere with native protein functions and could contribute to AML. However, exactly how the mutants exert their putative leukemogenic action remains elusive. Den Besten et $\mathrm{al}^{45}$ showed that NPM mutant proteins dislocate both wild-type NPM and the Arf protein into the cytoplasm, but were unable to demonstrate any transforming activity of the NPM mutants in NIH-3T3 and in Atm-null MEF cells. These findings suggest that NPM mutants could exert their transforming activity in a tissue-specific manner, an issue that needs to be addressed using appropriate animal models. Animal models show that AML may arise as result of the cooperative effect of different genetic lesions (eg, FLT3-ITD mutations in combination with molecular alterations, such PML-RAR alpha or AML1ETO fusion genes). ${ }^{1}$ We previously demonstrated that FLT3-ITD mutations in AML with normal karyotype occur twice as frequently in cases with NPM mutations as in those without. ${ }^{8}$ Consequently, a major focus of future studies should be to determine whether interactions between FLT3-ITD and NPM mutations contribute to the leukemogenesis. Although at present there is no evidence for the leukemogenic potential of NPM mutations, if it should be demonstrated in the future, then retargeting NPM mutants to their physiologic cell compartment (nucleoli) might be a potential therapeutic strategy. ${ }^{36,37}$ This paper clearly shows that, to be successful, retargeting has to overcome 2 obstacles, the new NES motif and the mutated tryptophan(s) at the mutant $\mathrm{C}$-terminus.

\section{Acknowledgments}

We are indebted to Roberta Pacini, Alessia Tabarrini, and Barbara Verducci for their skillfull technical assistance, and to Claudia Tibidò for her excellent secretarial assistance. We would also like to thank Dr Geraldine Anne Boyd for her help in editing this paper.

\section{References}

1. Gilliland DG, Jordan CT, Felix CA. The molecular basis of leukemia. Hematology. Washington, DC: American Society of Hematology Education Program Book; 2004:80-97.

2. Grimwade D, Walker H, Oliver F, et al. The importance of diagnostic cytogenetics on outcome in AML: analysis of 1,612 patients entered into the MRC AML 10 trial: the Medical Research Council Adult and Children's Leukaemia Working Parties. Blood. 1998;92:2322-2333.

3. Pabst T, Mueller BU, Zhang P, et al. Dominantnegative mutations of CEBPA, encoding CCAAT/ enhancer binding protein-alpha (C/EBPalpha), in acute myeloid leukemia. Nat Genet. 2001;27:263270.

4. Schnittger S, Schoch C, Dugas M, et al. Analysis of FLT3 length mutations in 1003 patients with acute myeloid leukemia: correlation to cytogenetics, FAB subtype, and prognosis in the AMLCG study and usefulness as a marker for the detection of minimal residual disease. Blood. 2002; 100:59-66.

5. Frohling S, Schlenk RF, Breitruck J, et al. Prognostic significance of activating FLT3 mutations in younger adults (16 to 60 years) with acute myeloid leukemia and normal cytogenetics: a study of the AML Study Group UIm. Blood. 2002;100: $4372-4380$.

6. Steudel C, Wermke M, Schaich M, et al. Comparative analysis of MLL partial tandem duplication and FLT3 internal tandem duplication mutations in 956 adult patients with acute myeloid leukemia. Genes Chromosomes Cancer. 2003; 37:237-251.

7. Bullinger L, Dohner K, Bair E, et al. Use of geneexpression profiling to identify prognostic subclasses in adult acute myeloid leukemia. N Engl J Med. 2004;350:1605-1616.

8. Falini B, Mecucci C, Tiacci E, et al. Cytoplasmic nucleophosmin in acute myelogenous leukemia with a normal karyotype. N Engl J Med. 2005; 352:254-266.

9. Colombo E, Marine JC, Danovi D, Falini B, Pelicci $P G$. Nucleophosmin regulates the stability and transcriptional activity of p53. Nat Cell Biol. 2002; 4:529-533.

10. Okuda M. The role of nucleophosmin in centrosome duplication. Oncogene. 2002;21:61706174.

11. Kurki S, Peltonen K, Latonen L, et al. Nucleolar protein NPM interacts with HDM2 and protects tumor suppressor protein p53 from HDM2-mediated degradation. Cancer Cell. 2004;5:465-475.

12. Bertwistle D, Sugimoto M, Sherr CJ. Physical and functional interactions of the Arf tumor suppressor protein with nucleophosmin/B23. Mol Cell Biol. 2004;24:985-996.

13. Brady SN, Yu Y, Maggi LB Jr, Weber JD. ARF impedes NPM/B23 shuttling in an Mdm2-sensitive tumor suppressor pathway. Mol Cell Biol. 2004; 24:9327-9338.

14. Korgaonkar C, Hagen J, Tompkins V, et al. Nucleophosmin (B23) targets ARF to nucleoli and inhibits its function. Mol Cell Biol. 2005;25:12581271.

15. Grisendi S, Bernardi R, Rossi M, et al. Role of nucleophosmin in embryonic development and tumorigenesis. Nature. 2005;437:147-153.

16. Borer RA, Lehner CF, Eppenberger HM, Nigg EA. Major nucleolar proteins shuttle between nucleus and cytoplasm. Cell. 1989;56:379-390.

17. Cordell JL, Pulford KA, Bigerna B, et al. Detection of normal and chimeric nucleophosmin in human cells. Blood. 1999;93:632-642.

18. Falini B, Mason DY. Proteins encoded by genes involved in chromosomal alterations in lymphoma 
and leukemia: clinical value of their detection by immunocytochemistry. Blood. 2002;99:409-426.

19. Lam YW, Trinkle-Mulcahy L, Lamond AI. The nucleolus. J Cell Sci. 2005;118:1335-1337.

20. Andersen JS, Lam YW, Leung AK, et al. Nucleolar proteome dynamics. Nature. 2005;433:77-83.

21. Alcalay M, Tiacci E, Bergomas R, et al. Acute myeloid leukemia bearing cytoplasmic nucleophosmin (NPMc+ AML) shows a distinct gene expression profile characterized by up-regulation of genes involved in stem-cell maintenance. Blood. 2005; 106:899-902.

22. Dohner K, Schlenk RF, Habdank M, et al. Mutant nucleophosmin (NPM1) predicts favorable prognosis in younger adults with acute myeloid leukemia and normal cytogenetics: interaction with other gene mutations. Blood. 2005;106:37403746 .

23. Schnittger S, Schoch C, Kern W, et al. Nucleophosmin gene mutations are predictors of favorable prognosis in acute myelogenous leukemia with a normal kayotype. Blood. 2005;106:37333739.

24. Verhaak RG, Goudswaard CS, van Putten W, et al. Mutations in nucleophosmin NPM1 in acute myeloid leukemia (AML): association with other gene abnormalities and previously established gene expression signatures and their favorable prognostic significance. Blood. 2005;106:37473754.

25. Gorello P, Cazzaniga G, Alberti F, et al. Quantitative assessment of minimal residual diseases in acute myeloid leukemia carrying nucleophosmin (NPM1) gene mutations. Leukemia. Prepublished on March 16, 2006, as DOI 10.1038/sj.leu.2404149.

26. Nakagawa M, Kameoka Y, Suzuki R. Nucleophosmin in acute myelogenous leukemia. $\mathrm{N}$ Engl J Med. 2005;352:1819-1820.

27. Quentmeier H, Martelli MP, Dirks WG, et al. Cell line OCI/AML3 bears exon-12 NPM gene mutation-A and cytoplasmic expression of nucleophosmin. Leukemia. 2005;19:1760-1767.
28. Schreiber E, Matthias P, Muller MM, Schaffner W. Rapid detection of octamer binding proteins with "mini-extracts", prepared from a small number of cells. Nucleic Acids Res. 1989;17:6419.

29. Cordell JL, Falini B, Erber WN, et al. Immunoenzymatic labeling of monoclonal antibodies using immune complexes of alkaline phosphatase and monoclonal anti-alkaline phosphatase (APAAP complexes). J Histochem Cytochem. 1984;32: 219-229.

30. Cazzaniga G, Dell'Oro MG, Mecucci C, et al. Nucleophosmin mutations in childhood acute myelogenous leukemia with normal karyotype. Blood. 2005;106:1419-1422.

31. Suzuki T, Kiyoi H, Ozeki K, et al. Clinical characteristics and prognostic implications of NPM1 mutations in acute myeloid leukemia. Blood. 2005; 106:2854-2861.

32. Boissel N, Renneville A, Biggio V, et al. Prevalence, clinical profile, and prognosis of NPM mutations in AML with normal karyotype. Blood. 2005;106:3618-3620.

33. Bogerd HP, Fridell RA, Benson RE, Hua J, Cullen BR. Protein sequence requirements for function of the human T-cell leukemia virus type 1 Rex nuclear export signal delineated by a novel in vivo randomization-selection assay. Mol Cell Biol. 1996;16:4207-4214.

34. la Cour T, Gupta R, Rapacki K, Skriver K, Poulsen FM, Brunak S. NESbase version 1.0: a database of nuclear export signals. Nucleic Acids Res. 2003;31:393-396.

35. Fukuda M, Asano S, Nakamura T, et al. CRM1 is responsible for intracellular transport mediated by the nuclear export signal. Nature. 1997;390:308311.

36. Macara IG. Transport into and out of the nucleus. Microbiol Mol Biol Rev. 2001;65:570-594.

37. Kau TR, Way JC, Silver PA. Nuclear transport and cancer: from mechanism to intervention. Nat Rev Cancer. 2004;4:106-117.

38. Koster M, Lykke-Andersen S, Elnakady YA, et al.
Ratjadones inhibit nuclear export by blocking CRM1/exportin 1. Exp Cell Res. 2003;286:321331.

39. Meissner T, Krause E, Vinkemeier U. Ratjadone and leptomycin B block CRM1-dependent nuclear export by identical mechanisms. FEBS Lett. 2004;576:27-30.

40. Wang W, Budhu A, Forgues M, Wang XW. Temporal and spatial control of nucleophosmin by the Ran-Crm1 complex in centrosome duplication. Nat Cell Biol. 2005;7:823-830.

41. Nishimura $Y$, Ohkubo T, Furuichi $Y$, Umekawa $H$. Tryptophans 286 and 288 in the $\mathrm{C}$-terminal region of protein B23.1 are important for its nucleolar localization. Biosci Biotechnol Biochem. 2002;66: 2239-2242.

42. Bischof D, Pulford K, Mason DY, Morris SW. Role of the nucleophosmin (NPM) portion of the nonHodgkin's lymphoma-associated NPM-anaplastic lymphoma kinase fusion protein in oncogenesis. Mol Cell Biol. 1997;17:2312-2325.

43. Falini B, Pulford K, Pucciarini A, et al. Lymphomas expressing ALK fusion protein(s) other than NPM-ALK. Blood. 1999;94:3509-3515.

44. Falini B, Bigerna B, Pucciarini A, et al. Aberrant subcellular expression of nucleophosmin and NPM-MLF1 fusion protein in acute myeloid leukaemia carrying $t(3 ; 5)$ : a comparison with NPMc+ AML. Leukemia. 2006;20:368-371.

45. den Besten W, Kuo ML, Williams RT, Sherr CJ. Myeloid leukemia-associated nucleophosmin mutants perturb p53-dependent and independent activities of the Arf tumor suppressor protein. Cell Cycle. 2005;4:1593-1598.

46. Daelemans D, Costes SV, Lockett S, Pavlakis GN. Kinetic and molecular analysis of nuclear export factor CRM1 association with its cargo in vivo. Mol Cell Biol. 2005;25:728-739.

47. Lobato MN, Rabbitts TH. Intracellular antibodies as specific reagents for functional ablation: future therapeutic molecules. Curr Mol Med. 2004;4: 519-528. 


\section{Clood \\ 2006 107: 4514-4523 \\ doi:10.1182/blood-2005-11-4745 originally published online \\ February 2, 2006}

\section{Both carboxy-terminus NES motif and mutated tryptophan(s) are crucial for aberrant nuclear export of nucleophosmin leukemic mutants in NPMc ${ }^{+}$AML}

Brunangelo Falini, Niccolò Bolli, Jing Shan, Maria Paola Martelli, Arcangelo Liso, Alessandra Pucciarini, Barbara Bigerna, Laura Pasqualucci, Roberta Mannucci, Roberto Rosati, Paolo Gorello, Daniela Diverio, Giovanni Roti, Enrico Tiacci, Giovanni Cazzaniga, Andrea Biondi, Suzanne Schnittger, Torsten Haferlach, Wolfgang Hiddemann, Massimo F. Martelli, Wei Gu, Cristina Mecucci and Ildo Nicoletti

Updated information and services can be found at: http://www.bloodjournal.org/content/107/11/4514.full.html

Articles on similar topics can be found in the following Blood collections

Free Research Articles (5281 articles)

Neoplasia (4182 articles)

Oncogenes and Tumor Suppressors (795 articles)

Information about reproducing this article in parts or in its entirety may be found online at:

http://www.bloodjournal.org/site/misc/rights.xhtml\#repub_requests

Information about ordering reprints may be found online at:

http://www.bloodjournal.org/site/misc/rights.xhtml\#reprints

Information about subscriptions and ASH membership may be found online at:

http://www.bloodjournal.org/site/subscriptions/index.xhtml

Blood (print ISSN 0006-4971, online ISSN 1528-0020), is published weekly by the American Society of Hematology, 2021 L St, NW, Suite 900, Washington DC 20036.

Copyright 2011 by The American Society of Hematology; all rights reserved. 